\title{
SALA DE ESPERA: UM ESPAÇO PARA O ENFERMEIRO EDUCAR CRIANÇAS COM FIBROSE CÍSTICA E SEUS FAMILIARES
}

\author{
WAITING ROOM: A NURSE TEACHING SPACE FOR CHILDREN \\ SUFFERING FROM CYSTIC FIBROSIS AND THEIR FAMILY MEMBERS \\ ANTESALA: UN LOCAL PARA EL ENFERMERO \\ EDUCAR NIÑOS CON FIBROSIS QUÍSTICA Y SUS FAMILIAS
}

\author{
Maria do Carmo Rocha Laurent* \\ Helena Becker Issi**
} * Enfermeira do Serviço de Enfermagem Pediátrica do Hospital de Clínicas de Porto Alegre, Unidade de Internação Pediátrica Sul. Responsável
pelo Programa de Assistência de Enfermagem à Criança com Fibrose Cística e seus Familiares.

** Enfermeira - Professora/Mestre - Escola de Enfermagem - Universidade Federal do Rio Grande do Sul - Chefe do Serviço de Enfermagem Pediátrica do Hospital de Clínicas de Porto Alegre.

\begin{abstract}
RESUMO. Revelando-se como abordagem inovadora, grupo de sala de espera constitui-se em recurso metodológico valioso no cuidado das crianças com Fibrose Cística (FC) e seus familiares. Este relato de experiência focaliza a atuação da enfermeira pediátrica no desenvolvimento desta abordagem pioneira em hospital universitário, referência nacional, no atendimento aos pacientes com FC. O objetivo é revelar as atividades adotadas pela enfermagem pediátrica para o trabalho com famílias e crianças em sala de espera. Reconhecendo a FC como doença crônica e progressiva, com manifestações clínicas numerosas, tornou-se fundamental que os profissionais de saúde preparassem estas crianças e suas famílias para o enfrentamento da doença. Estes grupos ocorrem semanalmente, por ocasião das consultas ambulatoriais, freqüentadas por estes pacientes a cada dois meses. A abordagem metodológica consiste em: acolhimento do paciente e seus familiares; explanação dos objetivos a que se propõe este espaço; levantamento de assuntos e/ou dúvidas a serem trabalhados a partir das expectativas do próprio grupo. Esta metodologia de cuidado enseja o convívio com outras famílias que vivem as mesmas dificuldades e diálogo livre com orientação de um profissional, constituindo-se em reforço contínuo dos cuidados em relação à doença. Visualiza-se o desenvolvimento desta prática como abordagem cuja essência expressa o acolhimento como estratégia para potenciar os recursos interiores tanto da família, quanto da criança, para o enfrentamento das mudanças existenciais desencadeadas pela doença crônica, hospitalizações freqüentes e tratamentos repetitivos e prolongados.
\end{abstract}

PALAVRAS-CHAVE: fibrose cística; criança; relação profissional; educação em saúde; família.

ABSTRACT. Introduced as an innovating approach, the waiting room group is a valuable methodological resource for caring children suffering from Cystic Fibrosis (CF) and their relatives. This experience report focus on the performance of the pediatrics nurse upon developing this pioneering approach in a university hospital that is national reference in treating patients with CF. This report purpose to disclose the activities adopted by the pediatrics nursing upon working with families and children in a waiting room. Recognizing being CF as a chronic and progressive disease with numerous clinic manifestations, it became fundamental that health professionals prepare these children and families to face the disease. Such groups meet weekly at the occasion of the patients' ambulatory consultations held each two months. The methodological approach consists of: welcoming the patients and their relatives; explanation of the space objectives; survey of issues and/or doubts to be worked by starting from the expectations of the group itself. This care methodology provides the opportunity of sharing with other families whose experiences the same difficulties as well as the free dialogue with orientation by a professional, becoming a continuous encouragement regarding the care vis-à-vis the disease. The development of this practice is regarded as an approach whose essence expresses welcoming as a strategy to strength the inner resources from both family and child in order to face the existential changes deriving from the chronic disease, frequent hospitalizations, repetitive and long-lasting treatments.

KEYWORDS: cystic fibrosis; child; professional relation; health education; family.

RESUMEN. Por revelarse como un abordaje innovador, grupo de antesala constituye un recurso metodológico valioso en el cuidado a niños con Fibrosis Quística (FQ) y a sus familias. Es un relato de experiencia que muestra la actuación de la enfermera pediátrica en el desarrollo de este abordaje pionero en un hospital universitario, referencia nacional para atender a pacientes con FQ. El objetivo es revelar las actividades adoptadas en la Enfermería pediátrica para trabajar con familias y niños en la antesala. Reconociendo la FQ como una enfermedad crónica y progresiva, con manifestaciones clínicas numerosas, se tornó fundamental que los profesionales de salud preparen a estos niños para el enfrentamiento de la enfermedad. Los grupos son semanales, debido a las consultas en el ambulatorio que frecuentan estes pacientes y sus familias a cada dos meses. El abordaje metodológico consiste en: acogida de los pacientes y de sus familias, colocación de los objetivos a que se propone este espacio, levantamiento de temas e/o dudas a ser trabajados a partir de las expectativas del propio grupo. Esta metodología de cuidado promueve la convivencia con otras familias que viven las mismas dificultades y diálogo libre con orientación profesional, constituyéndose en refuerzo continuo de los cuidados con relación a la enfermedad. El desarrollo de esta práctica se muestra como un abordaje que en su esencia promueve el acogimiento como una estrategia para potenciar los recursos interiores tanto de la familia, como del niño, para enfrentar los cambios existenciales desencadenados por la enfermedad crónica, hospitalizaciones frecuentes y tratamientos repetidos y prolongados.

PALABRAS-CLAVE: fibrosis quística; niño; relación profesional; educación en salud; familia.

Recebido em: 12/02/2005

Aceito em: 30/06/2005
Maria do Carmo Rocha Laurent

Rua Freire Alemão, 512/301A - Porto Alegre - RS

Fone: 3332-0971 - E-mail: carmolaurent@yahoo.com.br 


\section{INTRODUÇÃO}

Revelando-se como abordagem inovadora e expressiva por seu caráter facilitador à transformação de atitudes, um grupo de sala de espera constitui-se em recurso metodológico valioso para a prática de enfermagem no cuidado das crianças com fibrose cística e seus familiares, em unidade ambulatorial.

Este trabalho pretende focalizar a atuação da enfermeira pediátrica no desenvolvimento desta abordagem pioneira em nosso meio, desencadeada pela interdisciplinaridade e integração docente assistencial, característica de um hospital universitário, referência no atendimento a pacientes portadores de fibrose cística. Trata-se de um relato de experiência com o objetivo de revelar as atividades adotadas pela enfermagem pediátrica para o trabalho com famílias e crianças em grupos de sala de espera.

Há mais de duas décadas a Pediatria do Hospital de Clínicas de Porto Alegre (HCPA) vem recebendo crianças com fibrose cística. Com o passar dos anos e um número crescente destes pacientes, tornou-se indispensável a formação de uma equipe multidisciplinar para o atendimento destas crianças e de suas famílias. Hoje o HCPA é centro de referência nacional e latino americana para Fibrose Cística.

Defini-se fibrose cística (FC) como doença genética, autossômica recessiva, que se caracteriza por infecções respiratórias de repetição, tosse crônica, sibilância, baqueteamento digital, dificuldade de ganho ponderal, esteatorréia e excesso de sal no suor ${ }^{1}$. O tratamento da FC é contínuo e complexo, incluindo antibioticoterapia, fisioterapia e instituição de nutrição adequada ${ }^{2}$.

Os estudiosos apontam a fibrose cística (FC) como a causa mais comum de doença pulmonar supurativa crônica em brancos, no passado, era fatal no início da infância em conseqüência de bronquiectasias e insuficiência respiratória progressiva, mas com os avanços da antibioticoterapia e da nutrição, a sobrevida desses pacientes vem aumentando significativamente ${ }^{3}$.

Reconhecendo a fibrose cística como doença crônica e progressiva, com manifestações clínicas numerosas, torna-se fundamental que os profissionais de saúde preparem estas crianças e suas famílias para o enfrentamento da doença, possibilitando maior adesão ao tratamento.

A enfermagem pediátrica, reconhecendo suas potencialidades para conferir aos familiares melhores condições de cuidar e não esmorecer diante das dificuldades impostas em sua trajetória, deve implementar intervenções efetivas e eficazes no trabalho com famílias.

A enfermagem tem compromisso e obrigação de incluir as famílias nos cuidados de saúde. A evidência teórica, prática e investigacional do significado que a família dá para o bem-estar e a saúde de seus membros, bem como a influência sobre a doença, obriga as enfermeiras a considerar o cuidado centrado na família como parte integrante da prática de enfermagem. Entretanto este enfoque do cuidado pode ser alcançado somente com responsabilidade e respeito, estabelecendo-se práticas de avaliação e intervenção familiares confiáveis $4: 13$.

A criação do Programa: "Assistência de Enfermagem à Criança com Fibrose Cística e seus Familiares", constituindo-se em ações diferenciadas do enfermeiro no cotidiano do cuidado, dá margem à enfermeira para assumir a posição de referência não só para os pacientes e seus familiares como também para a equipe multidisciplinar.

$\mathrm{O}$ tratamento do paciente com FC preconiza internações sistemáticas e freqüentes durante toda a sua vida. Eles são internados nas exacerbações da doença, para tratamento das infecções pulmonares, por ocasião do diagnóstico, para avaliação da criança e orientação dos familiares, ou ainda quando há dificuldade de ganho ponderal para obter uma melhor nutrição ou quando existe dificuldades de adesão ao tratamento ${ }^{5}$.

Em cada internação o paciente é avaliado por todos os membros da equipe constituída por médicos, 
enfermeiros, nutricionistas, fisioterapeutas, psicólogos, assistentes sociais e pedagogos, com o objetivo de possibilitar que as crianças e seus familiares possam viver de modo muito próximo do normal, apesar da desvantagem que a doença crônica acarreta ${ }^{6}$.

Embora os pacientes com FC sejam internados de forma sistemática no decorrer de suas vidas, eles passam o maior tempo nas suas casas e devem tentar levar uma vida o mais normal possível. Portanto necessitam ser acompanhados e avaliados também neste período. Os pacientes com FC do HCPA têm consultas médicas cada 60 dias, avaliação da nutricionista e encaminhamento ao fisioterapeuta quando necessário e, desde a implantação do Programa "Assistência de Enfermagem à Criança com FC e seus Familiares", contam com orientações em um Grupo de Sala de Espera, realizado pela enfermeira.

Essa iniciativa vem somar esforços no sentido de ampliar as possibilidades da criança e da família de aprender a lidar com as vicissitudes impostas pela necessidade de conviver com uma doença crônica, cujo tratamento inclui estratégias de cuidado hospitalar, ambulatorial e domiciliar.

Para a equipe esta estreita e sensível convivência com a criança e, com a família fornece subsídios que emergem do processo dialógico que assim se estabelece, ensejando aproximação com a realidade vivenciada, através da ótica dos sujeitos do cuidado.

\section{A CONSTRUÇÃO DE UMA CAMINHADA NA ATENÇÃO DIFERENCIADA A CRIANÇA COM FC E AO CUIDADOR FAMILIAL}

Alguns fatores tornaram-se decisivos para que a Enfermagem Pediátrica acionasse o interesse particular e decisivo para a qualificação do cuidado às crianças com FC e seus familiares. Sensibilizada com os apelos constantes da equipe multidisciplinar de atendimento a esta clientela, especialmente por parte da equipe médica, que também antevia, na participação da enfermeira, a aquisição do mérito que a abordagem educativa teria, iniciaram os investimentos na aprimoração técnico-científica da enfermeira, com base no conhecimento vivencial que a práxis especializada do cuidado confere, articulada a outras iniciativas de desenvolvimento profissional.

A atuação da enfermeira como integrante da equipe multidisciplinar em internação pediátrica já se consolidava como marca decisiva de trabalho compartilhado em atenção às peculiaridades do acompanhamento na instituição do tratamento. Este, que em sua totalidade abrange as questões nutricionais, pulmonares, metabólicas, medicamentosas, entre outras, sem contar os aspectos peculiares que somente a sistematização da assistência de enfermagem pode alcançar, vai além das questões biológicas, alcançando razões psicossociais, espirituais e filosófico-existenciais, para as quais a enfermagem encontra-se direcionada a compreender, de modo abrangente.

Tal entendimento fundamentado na necessidade imperiosa de compreensão que os pais possuem dos momentos existenciais vividos e das decisões e atitudes que necessitam implementar para o cuidado cotidiano de suas crianças, impulsionou a criação de uma proposta de atuação em que a enfermagem não se limitasse ao atendimento em unidade de internação, mas que atingisse também o espaço de cuidado ambulatorial, freqüentado por esta clientela na continuidade de seus cuidados de saúde.

\section{FALANDO SOBRE SALA DE ESPERA}

Para a criação deste espaço e proposta metodológica de assistência articulada ao ensino e aprendizagem, foi necessária a realização de uma sondagem do contexto ambulatorial, enquanto ambiente do cuidado, que apontou:

- a média de atendimentos ambulatoriais é de 12 a 16 pacientes/dia;

- existe sala de espera específica para três consultórios de uso da equipe de pneumopediatria;

- os pacientes realizam consultas médicas, de nutrição e são encaminhados ao fisioterapeuta quando necessário; 
- vários pacientes e familiares permanecem durante muito tempo na sala de espera, aguardando consulta;

- os pacientes e familiares se conhecem e trocam informações naturalmente neste local;

- as dificuldades em realizar o tratamento adequadamente são muito semelhantes;

- os familiares demonstram necessidade de apoio cognitivo permanente.

Desse modo, a realização de grupos de sala de espera concretizou-se como mais uma estratégia de cuidado das famílias e crianças com FC, possibilitando espaço criativo e construtivo de saberes, pela interação entre clientes e enfermeira.

No trabalho, "Dialogar Saúde em Sala de Espera: Uma Proposta Multiprofissional", os autores ${ }^{7}$ abordam que, orientados por outra visão de saúde e de realidade, se concebeu Sala de Espera como um lócus propício para elaborar e socializar conhecimentos e/ou saberes, fundados em relações interpessoais politizadoras e menos autoritárias.

Ao referir-se a respeito do agir dialógico e problematizador salienta que não há modelo de como proceder, mas apenas pressupostos que podem indicar caminhos e que terão de ser constantemente refeitos ${ }^{8}$.

Valendo-se dessa posição, pode-se inferir que problematizar em Sala de Espera também é questão para a qual não há fórmulas preestabelecidas. É uma experiência que se constrói na medida em que usuários e profissionais, como sujeitos cognocentes, se criam e se recriam, constantemente, buscando sempre a superação.

A abordagem metodológica utilizada para 0 desenvolvimento dos grupos de sala de espera, consiste em:

- acolhimento do pacientes e seus familiares;

- explanação dos objetivos a que se propõe o trabalho realizado neste espaço;

- levantamento de assuntos e/ou dúvidas a serem trabalhados, a partir das expectativas do próprio grupo de crianças e/ou familiares;
- questionamento do profissional acerca de como vem sendo realizado o tratamento em casa;

- troca de experiências entre os participantes;

- propostas para mudança de comportamento em relação ao tratamento;

- fechamento e reforço pelo profissional;

- troca de informações pertinentes com os outros profissionais.

Essa metodologia de cuidado ocasiona o convívio com outras famílias que vivem as mesmas dificuldades; e o diálogo livre, com orientação de um profissional da área de saúde, fazendo um feedback, constituindo-se em reforço contínuo dos cuidados em relação à doença. Promove divulgação de novidades em relação ao tratamento e ao trabalho das Associações de Amparo à Fibrose Cística, atualizando os pacientes e suas famílias sobre assuntos pertinentes. É um momento peculiar para troca de experiências, de vivências e de sentimentos entre os paciente e as famílias. A visualização de pacientes de maior faixa etária com famílias adaptadas servem de estímulo às famílias ainda impactadas com o diagnóstico recente.

$\mathrm{O}$ respeito e a preocupação com a estética e a expressividade do cuidado são fundamentais na condução do processo dialógico que se estabelece em grupos de sala de espera.

O ser doente que habita o mundo do hospital necessita de compreensão, afeto e muito estímulo para assumir sua realidade existencial. A criança e a família, além da ação terapêutica, esperam da equipe de saúde compreensão, apoio e ajuda para enfrentar o cotidiano (...). A sensibilidade, o afeto, o sentimento de preocupação, a solidariedade e a cooperação, são elementos fundamentais, pontuados pela equipe como valores que devem reger as relações "com o outro" (...) 9:127.

Em análise de conteúdo ${ }^{10}$, realizada a partir de depoimentos colhidos com familiares e crianças em sala de espera, com objetivo de conhecer a ótica e a avaliação daqueles que vivenciam este 
contexto e estratégia de cuidado, revelou como tema principal: Sala de Espera: recurso facilitador no processo de enfrentamento da doença. As manifestações suscitadas podem ser explicitadas nos seguintes significados:

- Experiências de aprendizagem: reparo de enganos; procurar acertar; alcançar conhecimento;

- Sala de espera: espaço dialógico;

- Aceitação da doença e amadurecimento pessoal;

- Percepções e sentimentos reveladores de tranqüilidade, bem estar e gratidão, indicando uma avaliação positiva dos momentos vivenciados.

Tecendo uma retrospectiva dos grupos de sala de espera identificamos que os assuntos abordados se repetem ao longo do tempo. Baseados em levantamento dos assuntos trabalhados nos grupos de sala de espera podemos apresentar o seguinte:

- Em relação à doença: aspectos genéticos, fisiopatologia; manifestações clínicas; em relação ao diagnóstico: dificuldade de realizar o diagnóstico, teste do pezinho, eletrólitos no suor, impacto do diagnóstico, pesquisa genética.

- Em relação ao tratamento: fisioterapia, atividade física, alimentação, suplementação, enzimas pancreáticas, peso, medicações, controle periódico, internações, lista de internação, cuidados e desinfecção de nebulizadores, vacina da gripe, prevenção da infecção cruzada.

- Em relação ao enfretamento da doença: dificuldades na aceitação da doença, dificuldades na realização do tratamento, sentimentos, rotinas e disciplina (vivendo com FC), responsabilidade dos pais e da criança no tratamento.

- Em relação às complicações: uso de oxigênio no domicílio, gastrostomia e sonda nasoentérica, diabete na FC, desidratação na FC, transplante pulmonar, transplante hepático.

- Redes de apoio e outros enfoques: associações de amparo à $\mathrm{FC}$, escolas $\mathrm{e}$ creches, Secretaria da Saúde e distribuição de medicamentos, transferência para clínica de adultos, sexualidade na FC.

Ainda no ambulatório, percebe-se que outras atividades são desempenhadas pelo enfermeiro e incluem: a elaboração da lista de pacientes para internação, desenvolvimento de orientações préadmissionais e organização da agenda ambulatorial, a partir do levantamento da colonização dos pacientes e encaminhamentos a outros profissionais.

\section{CONSIDERAÇÕES FINAIS}

As dúvidas que afloram neste processo de conviver com uma doença crônica com tantas modificações ao viver que seria próprio da infância, acarretam profundo sofrimento que se traduz em sentimentos e percepções de insegurança, impotência, preocupações e até mesmo culpa.

Aprender a lidar com estas situações geradoras de sofrimento psicológico torna-se tão importante quanto administrar o processo terapêutico de forma adequada. Demandas psicológicas e de conhecimento técnico-científico são desafios que caminham lado a lado, e necessitam de abordagens articuladas de apoio emocional e cognitivo.

Grupos de sala de espera são processos de convivência mediante troca de experiências, de vivências e de sentimentos, pela oportunidade de diálogo orientado. Este espaço potencia o convívio de famílias com as mesmas dificuldades, reforço contínuo dos cuidados e esclarecimento de dúvidas em relação à doença, orientações pré-admissionais à internação pediátrica e socialização das novidades em relação ao tratamento e cuidados a que as crianças são submetidas.

Tais atividades contribuem para potenciar os recursos interiores, tanto da família quanto da criança, para o enfrentamento das mudanças existenciais 
desencadeadas pela doença crônica, hospitalizações freqüentes e tratamentos repetitivos e prolongados, que geram uma forma mais positiva de posicionaremse diante dos desafios que se impõem no viver cotidiano.

No processo de interação que assim se estabelece, a família pode vivenciar experiências de aprendizagem significativas, úteis não só durante o período de internação, mas transferíveis para a vida cotidiana fora do hospital, de modo a minimizar as dificuldades enfrentadas. Pode, igualmente, sentir-se valorizada, à medida que se percebe incluída nos programas educativos como participante, vendo reforçado seu compromisso em face do processo de cuidado dos filhos.

Nossa experiência aponta como benefícios dos grupos de sala de espera a adaptação das famílias, pelo estímulo mútuo; esclarecimento de dúvidas em relação à doença e a oportunidade de realizar um levantamento do conhecimento das crianças em relação à sua doença. Otimiza a interdisciplinaridade, na troca de informações e impressões do profissional da sala de espera com os profissionais dos consultório, possibilitando a discussão de casos e redução de custos, visto que o profissional atende maior número de pacientes no tempo em que eles "simplesmente" estariam esperando. Melhora a aceitação da doença e possibilita o preparo para a internação hospitalar. Com a implementação dos trabalhos no ambulatório por meio dos grupos de sala de espera, desenvolvidos semanalmente, percebe-se que esta metodologia funciona como reforço continuado para os pacientes e suas famílias.

Finalizando, fica a mensagem de que transcende à construção de cada etapa do trabalho criativo a certeza de que é imensurável seu valor como contribuição ao mundo vivido de cada familiar e de cada criança em particular, ao participar de tais atividades. Estes momentos de aprendizagem podem ser visualizados, como ingredientes para que novas compreensões possam ser alcançadas para o melhor cuidado de si e de outros, em busca de uma forma mais digna de viver e ser feliz.

\section{REFERÊNCIAS}

1 Kussek P, Gurmini J, Fernandes MGP, Lopes SM, Orlando ACT. Manual de fibrose cística. Curitiba: Hospital Pequeno Príncipe; 2005.

2 Grupo Brasileiro de Estudos em fibrose cística GBEFC. O que é fibrose cística. Disponível em: $<$ http://www. gbefc.org.br> (9 mai 2006).

3 Lissauer T, Cayden G. Manual ilustrado de pediatria. Rio de Janeiro: Guanabara Koogan; 1998.

4 Wright LM, Leahey M. Enfermeiras e famílias: um guia para avaliação e intervenção na família. São Paulo: Roca; 2002.

5 Wong DL. Enfermagem pediátrica: elementos essenciais à intervenção efetiva. Rio de Janeiro: Guanabara Koogan; 1999.

6 Scattolin I, Beier S, Blocher S, Laurent MCR, Santos MIS. Desenvolvimento da atenção integral à criança com fibrose cística. In: Ceccim RB, Carvalho, PRA, organizadores. Criança hospitalizada: atenção integral como escuta à vida. Porto Alegre: Editora da Universidade/ UFRGS; 1997. p.90-5.

7 Colomé CLM et al. Dialogar saúde em sala de espera: uma proposta multiprofissional. In: Programa de assistência integral da saúde da criança (PAISC). Hospital Universitário de Santa Maria (RS); 1991.

8 Siebert RS. Dominação e autoritarismo na prática pedagógica da educação física e as possibilidades de superação. Rev Educ Contexto 1991;(21).

9 Motta MGC. O ser doente no tríplice mundo da criança, família e hospital: uma descrição fenomenológica das mudanças existenciais. [tese]. Florianópolis (SC): Pós-graduação em Enfermagem da Universidade Federal de Santa Catarina; 1997.

10 Bardin LA. Análise de conteúdo. Lisboa: Edições 70; 2003. 